\title{
Formation mechanism of cracks in saturated sand
}

\author{
Xiaobing Lu • Zhemin Zheng • Yongren Wu
}

Received: 28 April 2005 / Revised: 8 November 2005 / Accepted: 20 February 2006 / Published online: 28 June 2006 (C) Springer-Verlag 2006

\begin{abstract}
The formation mechanism of "water film" (or crack) in saturated sand is analyzed theoretically and numerically. The theoretical analysis shows that there will be no stable "water film" in the saturated sand if the strength of the skeleton is zero and no positions are choked. It is shown by numerical simulation that stable water films initiate and grow if the choking state keeps unchanged once the fluid velocities decrease to zero in the liquefied sand column. The developments of "water film" based on the model presented in this paper are compared with experimental results.
\end{abstract}

Keywords Saturated sand · Cracks · Liquefaction

\section{Introduction}

It often occurs that the sand deposit on a slope translates to lateral spreading or even landslide not only during, but also after earthquakes. In the literature the possibility of existence of "water film" in sand containing an impermeable layer was first suggested by Seed [1] in his attempt to explain slope failure observed in earthquakes. The "water film" may serve as a sliding

The project supported by the National Natural Science Foundation of China (40025103 and 10202024) and Key Laboratory of Mountain Hazards and Surface Process, Chinese Academy of Sciences. The English text was polished by Keren Wang.

X. Lu $(\varangle) \cdot$ Z. Zheng · Y. Wu

Institute of Mechanics, Chinese Academy of Sciences,

Beijing 100080, China

e-mail: xblu@imech.ac.cn surface for postliquefaction failure. Therefore, landslide or debris flow may occur on a slope with a very moderate slope-angle. In the present paper we shall use the term crack instead of "water film". The crack in saturated sand is a water gap which forms when the permeability is nonuniform and therefore the pore water is trapped by relatively low permeable layers. The sand grains do not support one another and are therefore suspended in the condition of zero effective stresses [2]. The sand grains in suspension eventually settle down because they are heavier than water. The rate of such settlement is restricted by the fact that water must flow upward around the sand grains. If liquefiable sand deposits are overlaid by less permeable soils in a stratified or layered deposit, the overlaying deposit will restrict the pore water to pass through. If there is no downward drainage through the deposit, this relative flow at the interface, by continuity, must be equal to the velocity of settlement at the upper liquefied sand surface [3]. Thus water accumulates in the form of a water gap at the interface. This water gap is called "water film" or "crack". Feigel and Kutter [3] performed a centrifuge shake table test to demonstrate the formation of cracks in layered soils (The upper part is clay layer, the lower part is sand layer.). More recently, Kokusho [4] performed shake table tests using sand samples containing a seam of nonplastic silt and showed that cracks were formed under the silt layer. In this case the column was subjected to horizontal dynamic loadings to simulate earthquakes. Experimental observations on the formation of extended horizontal cracks in vertical columns of saturated sand contained in circular cylinders have also been reported by Zhang et al. [5] and Peng et al. [6]. In both cases, care is taken in preparing the sample by feeding 
wetted uniform sand continuously into a column of water to avoid inadvertent stratification. However, small inhomogenneity still exists due to uneven settling velocity.

These studies show that liquefaction is a necessary condition for crack initiation and growth. In Zhang's experiment, a sand column in a circular cylinder, whose porosity distribution is layered or has a perturbation in the vertical direction, is subjected to a vertical impact. It is found that small inhomogeneity of saturated sand may be aggravated during the settlement of the sand after liquefaction. The cracks appear at the position where fine grains accumulate (Fig. 1). It can be seen that some fine grains flow with the pore water upwards after liquefaction.

In the experiments of Peng et al. [6], the water is forced to percolate upward through the sand column in a circular cylinder whose porosity distribution is layered or has a perturbation in the vertical direction. The increase of this velocity is so slow that all inertia effects can be neglected. Liquefaction takes place locally as the flow rate is gradually raised. They find that such cracks appear only when the size of sand grains of the specimens varies in a broad range. Figure 2 is taken from Peng's [6] paper where $Y$ is the so-called liquefaction index defined as the ratio of the excess pore pressure to the effective stress. Liquefaction occurs when $Y$ is greater or equal to 1 . The points marked by a circle in Fig. 2 designate the position where a crack actually forms in the experiment. We note that in Fig. 2 the circled point lies on the line $Y=1$ which confirms the assertion that liquefaction is a necessary condition for the formation of a crack.

Cheng et al. [7] show analytically that the crack has to be just beneath the fine sand layer under some simple conditions. In their analysis, the porosity distribution is assumed to be periodic and the perturbation method is used to obtain the solutions.

The formation mechanism of cracks in a multilayered sand, especially, in a sand with the porosity distributed continuously is not very clear. This paper is a followup of the above studies. It discusses the progress on the study of the mechanism of the formation of such cracks.

Theoretical and numerical analyses are presented in this paper. First, a two-phase model describing the movement of liquefied sand is presented. While a full description of this process is not available, a simple empirical model is proposed to explain qualitatively the main features observed in experiments. Based on the model, some theoretical analysis is made on the conditions upon which the cracks are formed and the properties of the controlling equations. Numerical simulations

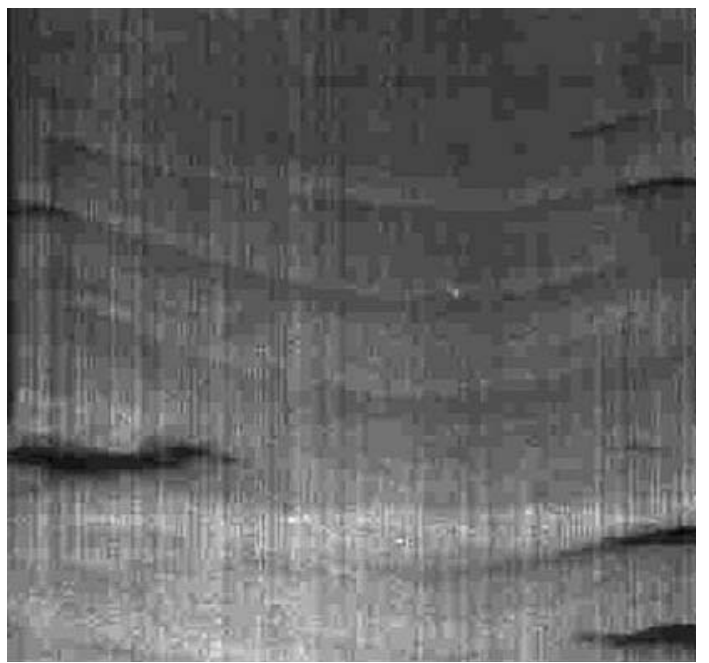

Fig. 1 The transverse cracks in a sand column (after [5])

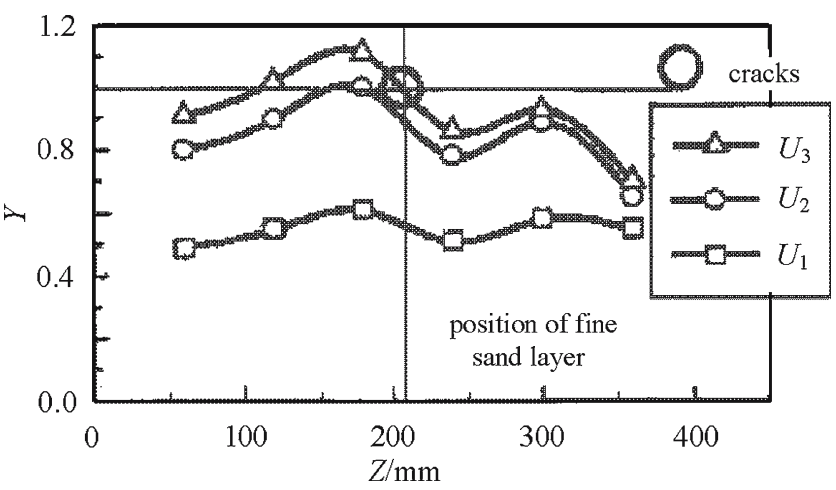

Fig. 2 The liquefaction indexes versus the depth (after [6]). The flow velocities are $U_{1}=0.08 \mathrm{~mm} / \mathrm{s}, U_{2}=0.13 \mathrm{~mm} / \mathrm{s}, U_{3}=0.16 \mathrm{~mm} / \mathrm{s}$

are then carried out to analyze the initiation, growth, and closure of the cracks.

\section{Formulation of the problem}

We consider a horizontal sand stratum which is water saturated and the porosity and other parameters change only in the vertical direction. The $x$ axis points upward (Fig. 3).

The following simplified two-phase flow equations are used. They are based on the assumptions that the flow is one-dimensional, the wall friction and the inertia effect may be neglected and only the simplest interaction between water and grains is considered. A broad grain size distribution means that some of the finer grains may be washed away to become part of the percolating fluid and, perhaps, be deposited later on somewhere down the stream, thus an initially homogeneous sand 


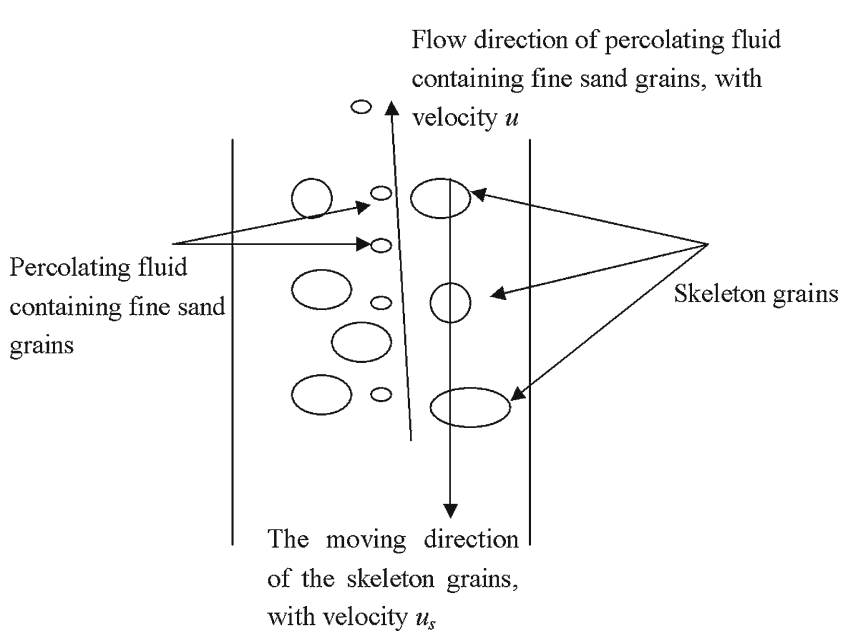

Fig. 3 The sketch of the model

column becoming inhomogeneous, that is, the permeability becoming inhomogeneous. This inhomogeneity can aggravate with time and with the increase of the flow rate and some place may be even choked because of the assembling of fine grains. The eroded sand mass is assumed to be limited by two factors: the eroded mass increases with the increase of the relative velocity and decreases with the increase of the fine sand mass in the fluid. Hence the answer to our question lies in properly describing the transport of these fine grains and its effect on permeability.

\subsection{Erosion relation}

In the experiment, when the grain size distribution of the sand is sufficiently narrow, cracks do not form. They form only when the grain size distribution is broad and there are fine grains. These observations show that the finer grains may be flushed away by the percolating water. This will have two consequences. First, the initial porosity will be altered. Second, the percolating water becomes turbid. Both factors lead to a modification of permeability in time and space.

It is assumed here that the sand transferred to water is proportional to the velocity difference of the grains and water, and the amount of sand which can be carried in the field is limited. Thus, the erosion relation is expressed as follows [7].

$$
\begin{gathered}
\frac{1}{\rho_{\mathrm{s}}}\left(\frac{\partial Q}{\partial t}+u_{\mathrm{s}} \frac{\partial Q}{\partial x}\right)=\frac{1}{T}\left(\frac{u-u_{\mathrm{s}}}{u^{*}}-q\right) \\
\text { if }-\varepsilon(x, 0) \leq \frac{Q}{\rho_{\mathrm{s}}} \leq \frac{Q_{\mathrm{c}}(x)}{\rho_{\mathrm{s}}}, \\
\frac{1}{\rho_{\mathrm{s}}}\left(\frac{\partial Q}{\partial t}+u_{\mathrm{s}} \frac{\partial Q}{\partial x}\right) \leq 0 \quad \text { otherwise, }
\end{gathered}
$$

in which the first term on the right side of the first equation shows how the sand is transferred to water, the second term describes that the deposition places a limit on the amount of sand that can be carried in the field, $Q$ is the mass of sand eroded per unit volume of the sand/water mixture, $\rho_{\mathrm{s}}$ is the density of the grains, $u$ and $u_{\mathrm{s}}$ are the velocities of the percolating fluid containing fine sand grains and the sand grains, $q$ is the volume fraction of sand carried in the percolating fluid, $T$ and $u^{*}$ are physical parameters, $\varepsilon(x, t)$ is the porosity, $Q_{\mathrm{c}}(x)$ is the maximum $Q$ that can be eroded at $x$.

\subsection{Conservation equations}

Considering the erosion of fine grains, the porosity is filled by two parts: one is the fine sand eroded from the skeleton, which is denoted by $q$ and another is the water, denoted by $\varepsilon-q$, and assuming that the fine sand flows with the water. The percolating fluid is composed of two parts: water $\varepsilon-q$ and eroded grains $q$. The mass conservation equations are as follows:

$$
\begin{aligned}
& \frac{\partial(\varepsilon-q) \rho}{\partial t}+\frac{\partial(\varepsilon-q) \rho u}{\partial x}=0, \\
& \frac{\partial q \rho_{\mathrm{s}}}{\partial t}+\frac{\partial q \rho_{\mathrm{s}} u}{\partial x}=G=\frac{\partial Q}{\partial t}+u_{\mathrm{s}} \frac{\partial Q}{\partial x}, \\
& \frac{\partial(1-\varepsilon) \rho_{\mathrm{s}}}{\partial t}+\frac{\partial(1-\varepsilon) \rho_{\mathrm{s}} u_{\mathrm{s}}}{\partial x}=-G=-\frac{\partial Q}{\partial t}-u_{\mathrm{s}} \frac{\partial Q}{\partial x},
\end{aligned}
$$

in which $\rho$ is the density of water, $G$ is the mass of fine grains eroded from the skeleton.

An integral equation may be obtained from the above three equations, which is

$\varepsilon u+(1-\varepsilon) u_{\mathrm{s}}=U(t)$,

in which $U(t)$ obviously is the flow rate of water per unit cross sectional area of the sand column.

\subsection{The momentum equilibrium equations}

The momentum equilibrium equations are as follows:

$$
\begin{aligned}
& {\left[(\varepsilon-q) \rho+q \rho_{\mathrm{s}}\right]\left(\frac{\partial u}{\partial t}+u \frac{\partial u}{\partial x}\right)=-\varepsilon \frac{\partial p}{\partial x}} \\
& \quad-\frac{\varepsilon^{2}\left(u-u_{\mathrm{s}}\right)}{k(\varepsilon, q)}-\left[(\varepsilon-q) \rho+q \rho_{\mathrm{s}}\right] g, \\
& {\left[(\varepsilon-q) \rho+q \rho_{\mathrm{s}}\right]\left(\frac{\partial u}{\partial t}+u \frac{\partial u}{\partial x}\right)} \\
& \quad+(1-\varepsilon) \rho_{\mathrm{s}}\left(\frac{\partial u_{\mathrm{s}}}{\partial t}+u_{\mathrm{s}} \frac{\partial u_{\mathrm{s}}}{\partial x}\right)=-\frac{\partial p}{\partial x}-\frac{\partial \sigma_{\mathrm{e}}}{\partial x} \\
& \quad-\left[(\varepsilon-q) \rho+q \rho_{\mathrm{s}}\right] g-(1-\varepsilon) \rho_{\mathrm{s}} g \\
& \quad-\left(\frac{\partial Q}{\partial t}+u_{\mathrm{s}} \frac{\partial Q}{\partial x}\right)\left(u-u_{\mathrm{s}}\right),
\end{aligned}
$$


in which Eq. (7) is the momentum equilibrium equation of percolating fluid, Eq. (8) is the total momentum equilibrium equation, the last term on the right side of Eq. (8) is the momentum of the eroded fine grains, $p$ is the pore pressure, $k$ is the physical permeability $\left(=K / \rho_{\mathrm{w}} g\right), \rho_{\mathrm{w}}$ is the density of the water. $K$ is the Darcy's permeability, $g$ is the gravity acceleration, $\sigma_{\mathrm{e}}$ is the effective stress. Here $k$ is assumed to be a function of $\varepsilon$ and $q$ in the following form [7]:

$k(\varepsilon, q)=k_{0} f(q, \varepsilon)=k_{0}(-\alpha q+\beta \varepsilon)$,

in which $\alpha, \beta$ are parameters and $1<\beta \ll \alpha$. We choose to let $\alpha$ much greater than $\beta$, so that changes in $q$ overweighs that of $\varepsilon$. In our analysis, two assumptions are adopted: (1) once the permeability $k$ at any place drops to zero, which means, choking occurs, the choking state keeps unchanged. (2) If any place is choked, the choking state may change, which means, the fluid may flow through this place again.

\subsection{Discontinuity relations and the conditions of cracks' formation}

Now, the expanding conditions of cracks are analyzed through the discontinuity. Assuming that the velocity of the discontinuity is $w$, translating the coordinate with the relation $\xi=x-w t$, as is often adopted in the analysis of discontinuity, e.g., shock wave [8], Eqs. (4)-(8) become

$$
\begin{aligned}
& \frac{\mathrm{d}}{\mathrm{d} \xi}(\varepsilon-q)(u-w)=0, \\
& \frac{\mathrm{d}}{\mathrm{d} \xi} q(u-w)=G, \\
& \frac{\mathrm{d}}{\mathrm{d} \xi}(1-\varepsilon)\left(u_{\mathrm{s}}-w\right)=-G, \\
& \frac{\mathrm{d}}{\mathrm{d} \xi}\left[(\varepsilon-q) \rho+q \rho_{\mathrm{s}}\right] u(u-w) \\
& \quad+\frac{\mathrm{d}}{\mathrm{d} \xi}(1-\varepsilon) \rho_{\mathrm{s}} u_{\mathrm{s}}\left(u_{\mathrm{s}}-w\right)+\frac{\mathrm{d}}{\mathrm{d} \xi}\left(p+\sigma_{\mathrm{e}}\right) \\
& =-\left[(\varepsilon-q) \rho+(1-\varepsilon+q) \rho_{\mathrm{s}}\right], \\
& \frac{1}{\varepsilon} \frac{\mathrm{d}}{\mathrm{d} \xi}\left[(\varepsilon-q) \rho+q \rho_{\mathrm{s}}\right] u(u-w)+\frac{\mathrm{d}}{\mathrm{d} \xi} p \\
& \quad=-\frac{H}{\varepsilon}-\left[\rho+\frac{q}{\varepsilon}\left(\rho_{\mathrm{s}}-\rho\right)\right],
\end{aligned}
$$

where $H$ is the so-called interactive force, which is assumed to be proportional to the velocity difference and $H=n^{2}\left(u-u_{\mathrm{s}}\right) / k[9], \sigma_{\mathrm{e}}$ is the effective stress of the skeleton.
By the above equations, five discontinuity relations may be obtained by integration at the discontinuity:

$$
\begin{aligned}
& \rho_{\mathrm{u}}^{+}=\rho^{-}, \\
& \left(\varepsilon^{+}-q^{+}\right)\left(u^{+}-w^{+}\right)=\left(\varepsilon^{-}-q^{-}\right)\left(u^{-}-w^{-}\right), \\
& \quad\left(1-\varepsilon^{+}\right)\left(u_{\mathrm{s}}^{+}-w^{+}\right)=\left(1-\varepsilon^{-}\right)\left(u_{\mathrm{s}}^{-}-w^{-}\right), \\
& \varepsilon^{+} \rho_{\mathrm{u}}^{+} u^{+}\left(u^{+}-w^{+}\right)+\left(1-\varepsilon^{+}\right) \rho_{\mathrm{s}}^{+} u_{\mathrm{s}}^{+}\left(u_{\mathrm{s}}^{+}-w^{+}\right) \\
& \quad+p^{+}+\sigma_{\mathrm{e}}^{+}=\varepsilon^{-} \rho_{\mathrm{u}}^{-} u^{-}\left(u^{-}-w^{-}\right) \\
& \quad+\left(1-\varepsilon^{-}\right) \rho_{\mathrm{s}}^{-} u_{\mathrm{s}}^{-}\left(u_{\mathrm{s}}^{-}-w^{-}\right)+p^{-}+\sigma_{\mathrm{e}}^{-}, \\
& \frac{1}{2} \rho_{\mathrm{u}}^{+}\left(u^{+}-w^{+}\right)^{2}+p^{+}=\frac{1}{2} \rho_{\mathrm{u}}^{-}\left(u^{-}-w^{-}\right)^{2}+p_{\mathrm{u}},
\end{aligned}
$$

in which $\rho_{\mathrm{u}}=\rho+q / \varepsilon\left(\rho_{\mathrm{s}}-\rho\right)$, and $q / \varepsilon$ and $\rho_{\mathrm{u}}$ are constants at the discontinuity. On the side of the water film $(\varepsilon=1)$, the velocity of water is $u_{1}$, the pore pressure is $p_{1}$, with no grains. Outside of the water film $(\varepsilon \neq 1)$, the related parameters include the porosity, the velocity of water, the pore pressure, the velocity of grains, and the effective stress of the skeleton $\varepsilon, u, p, u_{\mathrm{s}}, \sigma_{\mathrm{e}}$. Let $u-u_{1}=U, w-u_{1}=W, W$ is the velocity of discontinuity relative to the water film.

Then we can obtain the following relations according to the discontinuity relations

$$
\begin{aligned}
& u_{\mathrm{s}}=w \quad \text { or } \quad u_{\mathrm{s}}-u_{1}=W \text {, } \\
& \varepsilon U+(1-\varepsilon) W=0 \text {, } \\
& \Delta p=p_{1}-p=\frac{1}{2} \rho_{\mathrm{u}}\left[(U-W)^{2}-W^{2}\right] \\
& =\frac{1}{2} \frac{1-\varepsilon^{2}}{\varepsilon^{2}} \rho_{\mathrm{u}} W \text {, } \\
& \Delta p-\sigma_{\mathrm{e}}=-\rho_{\mathrm{u}} W U=\frac{1-\varepsilon}{\varepsilon} \rho_{\mathrm{u}} W^{2}, \\
& \sigma_{\mathrm{e}}=\frac{1}{2} \rho_{\mathrm{u}} U^{2}=\frac{1}{2} \frac{(1-\varepsilon)^{2}}{\varepsilon^{2}} \rho_{\mathrm{u}} W^{2} \text {. }
\end{aligned}
$$

It is clear that if $\sigma_{\mathrm{e}}$ is equal to zero, $W$ and $U$ are both equal to zero, which means that there are no cracks or stable cracks existing in this case. The formation of stable or expanding cracks requires that the effective stress is greater than zero or other conditions exist, such as the choking in the sand column.

\section{Numerical simulations}

3.1 Simplification of the equations and the characteristics of these equations

Based on the model presented above, we will analyze the initiation and growth of cracks in the saturated sand in the case about cracks in a liquefied sand $\left(\sigma_{\mathrm{e}}=0, \tau \approx 0\right)$ where the grains sink while the water and the fine grains eroded from the skeleton move upward. The above equations may be simplified, and some characteristics may be obtained.

Assume that both $u$ and $u_{\mathrm{s}}$ are zero at $x=0$, the total mass flow rate is

$\varepsilon u+(1-\varepsilon) u_{\mathrm{s}}=U(t)=0$. 
With Eqs. (1), (2), (9), and (13), Eqs. (4), (5), (7), and (8) can be reduced to

$$
\begin{aligned}
& \frac{\partial \varepsilon}{\partial t}+\frac{\partial u \varepsilon}{\partial x}=\frac{1}{T}\left[\frac{u}{u^{*}(1-\varepsilon)}-q\right], \\
& \frac{\partial q}{\partial t}+\frac{\partial u q}{\partial x}=\frac{1}{T}\left[\frac{u}{u^{*}(1-\varepsilon)}-q\right], \\
& \frac{1-\varepsilon}{\varepsilon}\left[(\varepsilon-q) \frac{\rho}{\rho_{\mathrm{s}}}+q\right]\left(\frac{\partial u}{\partial t}+u \frac{\partial u}{\partial x}\right) \\
& \quad+\varepsilon\left(\frac{\partial u}{\partial t}-u \frac{\varepsilon}{1-\varepsilon} \frac{\partial u}{\partial x}\right)+\frac{u}{1-\varepsilon}\left(\frac{\partial \varepsilon}{\partial t}-u \frac{\varepsilon}{1-\varepsilon} \frac{\partial \varepsilon}{\partial x}\right) \\
& =-\frac{\varepsilon u}{1-\varepsilon} \frac{1}{k_{0} \rho_{\mathrm{s}} f(q, \varepsilon)}-\frac{u}{\varepsilon(1-\varepsilon)}\left(\frac{u}{T u^{*}(1-\varepsilon)}-q\right) \\
& \quad+\frac{1-\varepsilon}{\varepsilon}(\varepsilon-q)\left(1-\frac{\rho}{\rho_{\mathrm{s}}}\right) g .
\end{aligned}
$$

Equation (14) can be further simplified by the observation that the crack formation is a slow and late stage process during which the inertia effect is very small. Since the erosion/re-deposition is considered as a controlling factor, it is reasonable to take $T$ in Eq. (14) as the appropriate characteristic time. Let $u_{\mathrm{t}}$ denote the characteristic velocity and $L$ the characteristic length of the problem. We shall make them more specific in the subsequent discussion. For the time being we use them to make Eq. (14) nondimensional. Letting

$\bar{u}=\frac{u}{u_{\mathrm{t}}}, \quad \tau=\frac{t}{T}, \quad \xi=\frac{x}{L}$,

the first two equations of Eqs. (14) become

$\frac{\partial \varepsilon}{\partial \tau}+\frac{T u_{\mathrm{t}}}{L} \frac{\partial \varepsilon \bar{u}}{\partial \xi}=\bar{u} \frac{u_{\mathrm{t}}}{u^{*}(1-\varepsilon)}-q$,

$\frac{\partial q}{\partial \tau}+\frac{T u_{\mathrm{t}}}{L} \frac{\partial q \bar{u}}{\partial \xi}=\bar{u} \frac{u_{\mathrm{t}}}{u^{*}(1-\varepsilon)}-q$.

When $T g / u_{\mathrm{t}} \gg 1$, the inertia terms are negligible and the last equation of Eq. (14) becomes

$$
\begin{aligned}
\bar{u} & =\left(\frac{1-\varepsilon}{\varepsilon}\right)^{2}(\varepsilon-q) f(q, \varepsilon) \frac{k_{0} \rho_{\mathrm{s}} g\left(1-\rho / \rho_{\mathrm{s}}\right)}{u_{\mathrm{t}}} \\
& =\left(\frac{1-\varepsilon}{\varepsilon}\right)^{2}(\varepsilon-q) f(q, \varepsilon),
\end{aligned}
$$

when $u_{\mathrm{t}}$ is taken to be

$u_{\mathrm{t}}=k_{0} \rho_{\mathrm{s}} g\left(1-\rho / \rho_{\mathrm{s}}\right)$.

Thus, aside from a constant factor of the order of one, $u_{\mathrm{t}} /(1-\varepsilon)$ is the settling velocity of sand grains in a uniform column of sand with constant permeability $k_{0}$. The problem now reduces to finding $\varepsilon(\xi, \tau)$ and $q(\xi, \tau)$ as a set of solutions to Eq. (16). The initial conditions are

$\varepsilon(\xi, 0)=\varepsilon_{0}(\xi), \quad q(\xi, 0)=0$.

In order to de-couple the problem from the complication arising from the effect of the consolidation wave initiated from the bottom of the sand column, it is assumed that the sand column is very tall so that cracks would develop before the consolidation wave arrives.

In order to obtain a full solution we rewrite Eq. (16) as

$$
\begin{aligned}
& \frac{\partial \varepsilon}{\partial \tau}+\frac{\partial \overline{u \varepsilon}}{\partial \zeta}=\bar{u} \frac{u_{\mathrm{t}}}{u^{*}(1-\varepsilon)}-q, \\
& \frac{\partial q}{\partial \tau}+\frac{\partial \overline{u q}}{\partial \varsigma}=\bar{u} \frac{u_{\mathrm{t}}}{u^{*}(1-\varepsilon)}-q .
\end{aligned}
$$

It can be shown that these equations are hyperbolic and the characteristic equations are given by

$$
\begin{aligned}
& \frac{\mathrm{d} \xi}{\mathrm{d} \tau}=\bar{u}+\varepsilon \frac{\partial \bar{u}}{\partial \varepsilon}+q \frac{\partial \bar{u}}{\partial q} \text { for } \mathrm{C}^{-} \text {characteristic, } \\
& \frac{\mathrm{d} \xi}{\mathrm{d} \tau}=\bar{u} \text { for } \mathrm{C}^{+} \text {characteristic }
\end{aligned}
$$

Along the $\mathrm{C}^{-}$and $\mathrm{C}^{+}$characteristics, $\varepsilon$ and $q$ satisfy, respectively, the following equations:

$$
\begin{aligned}
& \frac{\mathrm{d} \varepsilon}{\mathrm{d} \tau}+\left(\frac{\partial \bar{u}}{\partial q} / \frac{\partial \bar{u}}{\partial \varepsilon}\right) \frac{\mathrm{d} q}{\mathrm{~d} \tau}=\left(1+\frac{\partial \bar{u}}{\partial q} / \frac{\partial \bar{u}}{\partial \varepsilon}\right)\left(\frac{\lambda}{1-\varepsilon} \bar{u}-q\right), \\
& \frac{\mathrm{d} \varepsilon}{\mathrm{d} \tau}-\frac{\varepsilon}{q} \frac{\mathrm{d} q}{\mathrm{~d} \tau}=\left(1-\frac{\varepsilon}{q}\right)\left(\frac{\lambda}{1-\varepsilon} \bar{u}-q\right) .
\end{aligned}
$$

The above results show that the solution is completely determined when the value of $\varepsilon$ and $q$ are prescribed at $t=0$ for $-\infty<\xi<\infty$.

\subsection{Numerical results}

In this section, we will use a finite difference scheme to solve Eq. (20) under four initial conditions. The initial conditions are nonuniform and continuous distribution of porosity, three layered sand with different porosities in each layer which are similar to the conditions in the experiments of Zhang et al. [5], Peng et al. [6], and Kokusho and Kojima [10]. To each porosity distribution, two assumptions (the choking assumption and no choking state) are adopted, respectively: these different distributions of porosity are adopted to investigate the conditions that cracks may initiate, grow and close.

The parameters adopted in the calculation are as follows:

Material parameters: $\beta=47 \sim 56, \rho_{\mathrm{s}}=2,400 \mathrm{~kg} / \mathrm{m}^{3}$, $\rho_{\mathrm{W}}=1,000 \mathrm{~kg} / \mathrm{m}^{3}, u^{*}=0.04, k_{0}=4 \times 10^{-10} \mathrm{~m}^{3} \mathrm{~s} / \mathrm{kg}$, $\alpha=1$.

The parameters in the initial conditions: $\kappa=50.0$, $a=0.08$.

The time step: $\mathrm{d} \tau=9 \times 10^{-4}$

The computing results under four conditions are as follows: 
1. The initial porosity changes continuously, and it is assumed that once a place is choked, the choked state keeps unchanged. The porosity is in the form of $\varepsilon_{0}(x)=\bar{\varepsilon}_{0}(1-a \tanh ((\varsigma-0.5 L) / 2) \kappa)$, in which $\bar{\varepsilon}_{0}=0.4, L=1,0 \leq \varsigma \leq 1.6, L$ is the length of the computing zone. It is shown that the crack will initiate and grow gradually in this case, as shown in Fig. 4. The reason is that the sand above the choked position will be prevented from dropping across the choked position and so the porosity becomes smaller and smaller, while the sand below the positions will settle down gradually and make the crack initiate and grow gradually.

2. The porosity distribution is the same as that in condition (1), but with no choking assumption as in condition (1). It is shown that the crack will form first and then disappear gradually in this case (Fig. 5). It is because the choked position is dredged gradually.

3. The sand column consists of three layers. The upper part is dense sand with $\varepsilon_{0}=0.3$, the middle part is dense sand with $\varepsilon_{0}=0.2$ and the thickness is $L / 50$, the lower part is loose sand with $\varepsilon_{0}=0.4$. The assumption that $u$ keeps unchanged once it drops to zero, the same as in condition (1) is adopted. It is shown that there is a stable crack forming in this case (Fig. 6). The reason is similar to that in condition (1). The upper part and the middle part have smaller permeability than the lower part. Thus, the water percolating from the lower part cannot pass through the two overlayers and will accumulate in the interface between the lower part and the middle part.

4. The distribution of initial porosity is the same as that under condition (3), but without any assumptions. It is shown that there are no stable cracks forming in this case (Fig. 7). A crack forms first but closes gradually.

The above results show that nonuniformity of grain size distribution along the depth of the sand column is an essential precondition for cracks to initiate and grow. The transport of sand composed of fine grains by percolation tends to aggravate this nonuniformity. The porosity of the upper part must be smaller than that of the lower part for cracks to form. Liquefaction is a necessary condition for the formation of cracks.

\subsection{Comparison with the experimental results}

The results are compared with the experimental data of Kokusho and Kojima[10] (Fig. 8). In Kokusho's experiment, a saturated loose sand layer of $200 \mathrm{~cm}$ depth sandwiches a seam of nonplastic silt in the middle $(96 \mathrm{~cm}$

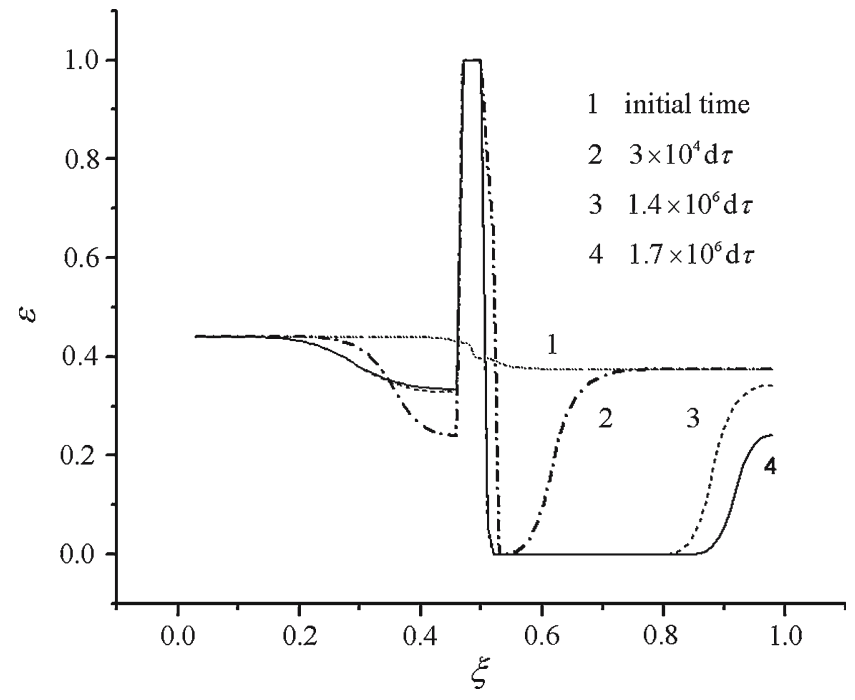

Fig. 4 The evolution of cracks in the initial and boundary condition 1

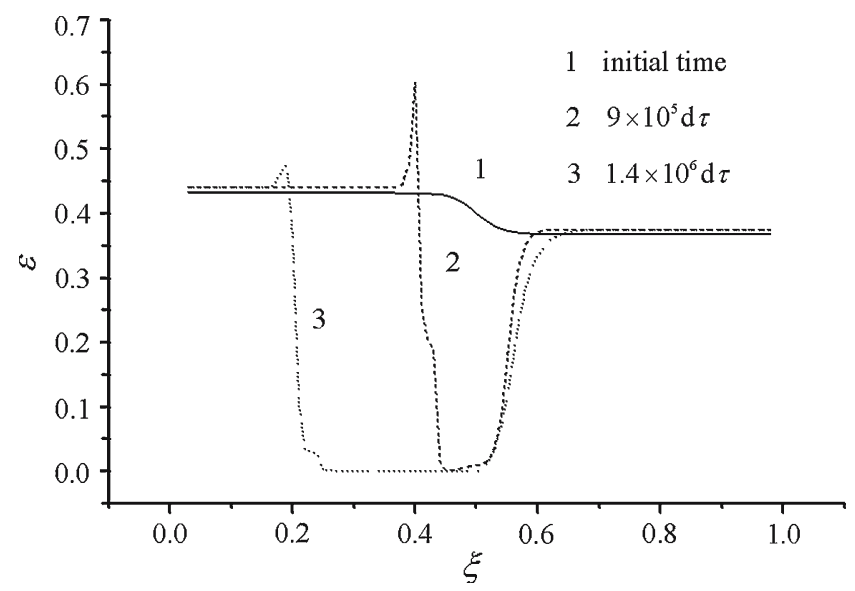

Fig. 5 The evolution of cracks in the initial and boundary condition 2

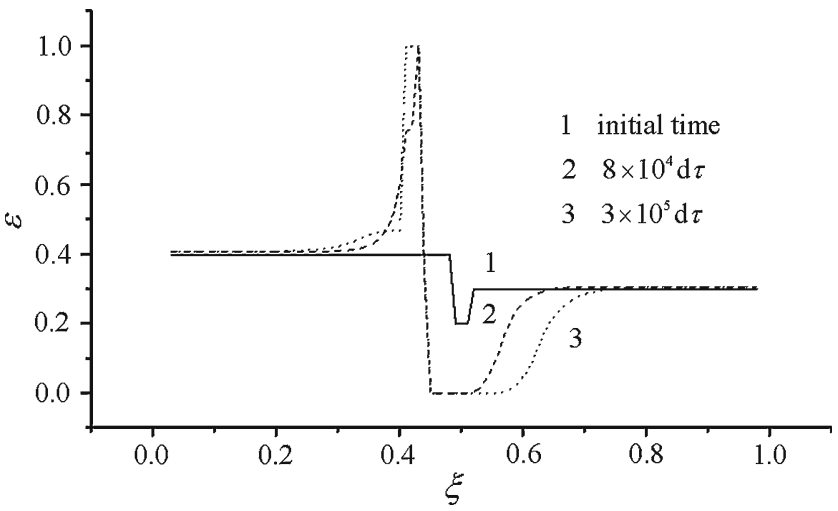

Fig. 6 The evolution of cracks in the initial and boundary condition 5 
Table 1 Parameters in literature of Kokusho and Kojima [10]

\begin{tabular}{llllll}
\hline Sand & Thickness $(\mathrm{cm})$ & Relative density $(\%)$ & Permeability $(\mathrm{cm} / \mathrm{s})$ & Initial porosity & Maximum of strain $(\%)$ \\
\hline Upper layer sand & 103.6 & 14 & 0.04 & 0.48 & 2.4 \\
Sandwich sand & 0.4 & & $1.8 \times 10^{-4}$ & 0.6 & 2.4 \\
Lower layer sand & 96 & 39 & 0.04 & 0.454 & 0.95 \\
\hline
\end{tabular}

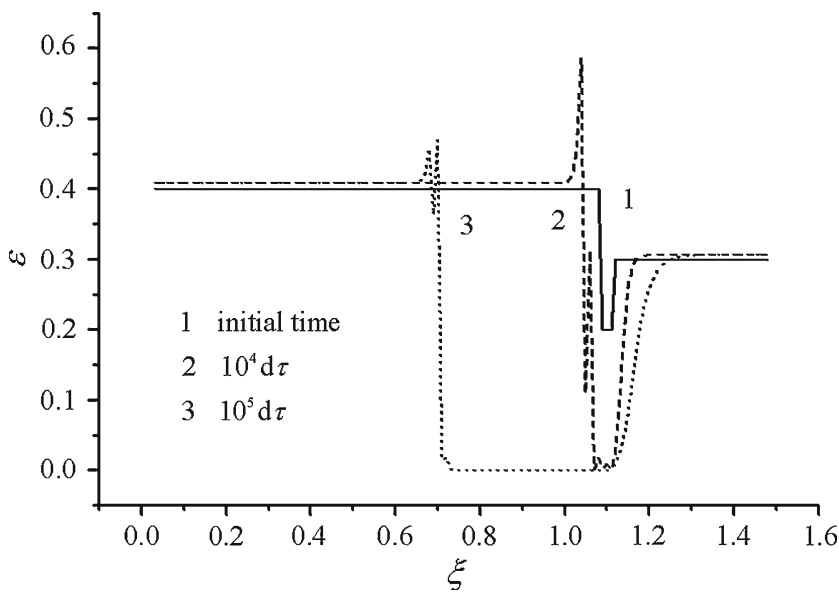

Fig. 7 The evolution of cracks in the initial and boundary condition 6

from the bottom). The initial void ratios of the upper, the middle, and the lower sand layers are $0.924,1.5$, and 0.831 , respectively. The initial permeability of the upper, the middle and the lower sand layers are 0.04 , 0.00018 , and $0.04 \mathrm{~cm} / \mathrm{s}$, respectively. The thickness of silt seam is about $4 \mathrm{~mm}$. The saturated sand is in a tube of $13 \mathrm{~cm}$ inner diameter and $211.5 \mathrm{~cm}$ height. The onedimensional sand layer is instantaneously liquefied by a loading with a steel hammer. In the comparison, we adopt the same data as that given in literature of Kokusho's [10](Table 1). In Fig. 8, we can see that the two results agree with each other to some extent. Here it is assumed that if the porosity is $10 \%$ bigger than the initial porosity, a crack comes into being.

\section{Conclusions}

The theoretical and numerical analysis show that there will be no stable crack in a saturated sand if there exists no effective stress and no choking occurs in the sand column. By numerical simulations, it is shown that nonuniformity of grain size distribution along the depth of the sand column is an essential precondition for cracks to initiate and grow. The transport of sand composed of

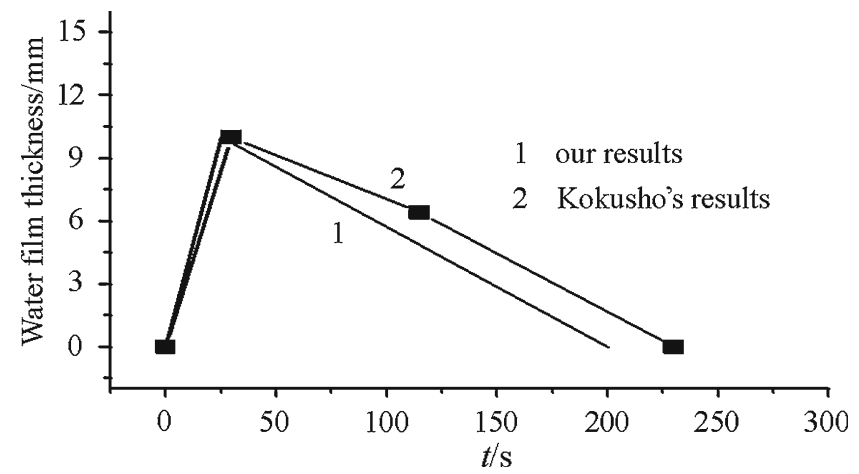

Fig. 8 The comparison of our results with that of Kokusho and Kojima [10]

fine grains by percolation tends to aggravate this nonuniformity. Liquefaction is a necessary condition for the formation of cracks.

\section{References}

1. Seed, H.B.: Design problems in sand liquefaction. J. Geotech. Engrg. ASCE 113(8), 827-845 (1987)

2. Scotter, R.F.: Solidification and consolidation of a liquefied sand column. Soils Found. 26(4), 23-31 (1986)

3. Fiegel, G.L., Kutter, B.L.: Liquefaction mechanism for layered sands. J. Geotech. Engrg. ASCE 120(4), 737-755 (1994)

4. Kokusho, T.: Water film in liquefied sand and its effect on lateral spread. J. Geotech. Geoenviron. Engrg. 10, 817-826 (1999)

5. Zhang, J.F., Meng, X.Y., Yu, S.B. et al.: Experimental study on permeability and settlement of saturated sand under impact loading. Acta. Mech. Sin. 312, 230-237 (1999) (in Chinese)

6. Peng, F.J., Tan, Q.M., Cheng, C.M.: Laboratory study on cracks in saturated sands. Acta. Mech. Sin. 16(1): 48-54 (2001)

7. Cheng, C.M., Tan, Q.M., Peng, F.J.: On the mechanism of the formation of horizontal cracks in a vertical column of saturated sand. Acta. Mech. Sin. 17(1), 1-9 (2001)

8. Courant, R., Friedrichs, K.O.: Supersonic Flow and Shock Waves, pp. 121-126, Interscience Publishers Inc. New York (1948)

9. Biot, M.A.: Theory of propagation of elastic waves in a fluidsaturated porous solid. In: Low frequency range. J. Acoust. Soc. Am. 28, 168-191 (1956)

10. Kokusho, T., Kojima T.: Mechanism for postliquefaction water film generation in layered sand. J. Geotech. Geoenviron ASCE 128(2), 129-137 (2002) 